\title{
Characterization of the Isoflavone Pratensein as a Novel Transcriptional Up-Regulator of Scavenger Receptor Class B Type I in HepG2 Cells
}

\author{
Yuan YANG, ${ }^{\#}$ Wei JIANG, ${ }^{\#}$ Li Wang, Zhong-Bing Zhang, Shu-Yi Si, ${ }^{*}$ and Bin Hong* \\ Institute of Medicinal Biotechnology, Chinese Academy of Medical Sciences \& Peking Union Medical College; Beijing \\ 100050, China. Received January 22, 2009; accepted April 9, 2009; published online April 15, 2009
}

\begin{abstract}
Scavenger receptor class B type I (SR-BI), as well as its human homologue CLA-1, plays an important role in reverse cholesterol transport (RCT) as high density lipoprotein (HDL) receptor. Using a previously developed cell-based screening model for CLA-1 up-regulators, pratensein, was shown to present activity in elevating CLA1 transcriptional level. In this study, three other isoflavones including formononetin, genistein and daidzein were also shown to up-regulate CLA-1 transcriptional activity in the cell-based reporter assay. The effects of pratensein on up-regulating CLA-1 expression were demonstrated at both mRNA and protein levels, and validated by its increasing of 1,1'-dioctadecyl-3,3,3',3'-tetramethylindocarbocyanine perchlorate-labeled (DiI)-HDL uptake in HepG2 cells. Furthermore, the cis-elements responsible for the pratensein up-regulatory effects were mapped to the $-1055 /-182$ bp fragment of CLA-1 promoter in HepG2 cells. These findings might provide a new molecular mechanism by which isoflavones potentially prevent atherosclerosis.
\end{abstract}

Key words pratensein; scavenger receptor class B type I; reverse cholesterol transport; atherosclerosis; cis-element

Cardiovascular disease is one of the most common diseases that damage human health in developed countries and most developing countries, while atherosclerosis is the principal pathogenesis for many critical cardiovascular diseases. Recent studies revealed that the antiatherosclerotic effect of high density lipoprotein (HDL) was mainly attributed to its role in reverse cholesterol transport (RCT). Scavenger receptor class B type I (SR-BI) is the first high-affinity HDL receptor to be identified at the molecular level, ${ }^{1)}$ and the human homologue of SR-BI was initially identified as CLA-1 (CD36 and lysosomal integral membrane protein-II analogous-1) and mapped to chromosome $12 .^{2)}$ The CLA-1/SR-BI receptor is abundantly expressed mainly in the liver, ${ }^{3)}$ where it mediates selective uptake of cholesteryl ester from HDL. It is also expressed in macrophages and can promote cholesterol efflux to HDL in vitro. ${ }^{4)}$ So CLA-1/SR-BI plays a pivotal role both in the initial (HDL-C efflux and removal from the artery wall) and final (selective HDL-C uptake in the liver) phase of RCT.

Phytoestrogens are plant-derived compounds that structurally or functionally mimic mammalian estrogens and therefore are considered to play an important role in the prevention of cancer, menopausal symptoms, osteoporosis, cardiovascular disease and atherosclerosis., ${ }^{5,6)}$ Isoflavones are the most important type of phytoestrogens found in legume plants such as soy (Glycine max L.) and red clover (Trifolium pratense L.). Potential antiatherogenic effects of isoflavones include: a reduction in low density lipoprotein (LDL) cholesterol; modulation of pro-inflammatory cytokines, cell adhesion proteins and nitric oxide $(\mathrm{NO})$ formation; protection of LDL against oxidation; inhibition of platelet aggregation; and an improvement in vascular reactivity, etc. (see Cassidy et al. $^{7)}$ and references therein). In a previous study, we found that an active compound 4776B may up-regulate CLA-1 expression in human hepatoma cell line (HepG2) cells using a developed cell-based reporter assay. ${ }^{8)}$ The chemical structure of $4776 \mathrm{~B}$ from an actinomycete strain was identified as pratensein, which is one of the isoflavone components in the leguminous plant $T$. pratense L. Red clover isoflavones have been extracted and commercialized as nutraceuticals, which is beneficial in post-menopausal women for bone, cardiovascular risk and hot flashes. ${ }^{9)}$ The hypolipidemic activity of pratensein has been reported in Triton-WR1339-induced hyperlipidemia in male albino rats. ${ }^{10)}$ However, the detailed mechanisms of pratensein have not been illuminated. In the previous study, pratensein was shown to up-regulate CLA-1 expression in HepG2 cells by some primary studies. ${ }^{8)}$ The aim of the present study was to investigate whether other isoflavones have similar activity, and to characterize the effects of pratensein on the key cellular transmembrane protein CLA-1/SR-BI which participates in RCT. Furthermore, we identified the cis-elements responsible for the up-regulation of CLA-1 expression in HepG2 cells by pratensein. These results might provide a new mechanism for the antiatherosclerotic activity of isoflavones.

\section{MATERIALS AND METHODS}

Materials Pratensein (4776B) was isolated from actinomycete $04-4776$ fermentation broth $\left(>95 \%\right.$ purity) ${ }^{8}{ }^{8}$ Formononetin, genistein and daidzein were from the pure chemical library of Institute of Medicinal Biotechnology (Chinese Academy of Medical Sciences, Beijing, China). 1,1'-Dioctadecyl-3,3,3',3'-tetramethylindocarbocyanine perchloratelabeled HDL (DiI-HDL) was purchased from Biomedical Technologies (Stoughton, MA, U.S.A.). White-wall, clear bottom 96-well polystyrene assay plates were obtained from BMG LABTECH (Offenburg, Germany); 96-well, 24-well and 6-well plates for cell culture were obtained from Corning (Corning, MA, U.S.A.). Cell culture media and fetal bovine serum (FBS) were from Hyclone (Logan, UT, U.S.A.). G418 was obtained from Invitrogen (Carlsbad, CA, U.S.A.).

Cell Culture HepG2 human hepatoma cells were grown in minimum essential medium with Earle's balanced salt and $2 \mathrm{mM}$ L-glutamine solution (MEM-EBSS) supplemented with $10 \% \mathrm{FBS}$ at $37^{\circ} \mathrm{C}$ in a humidified $5 \% \mathrm{CO}_{2}$ incubator. CLApLUC HepG2 cells, ${ }^{8)}$ which are HepG2 cells stably transfected with a gene construct in which the promoter and regulatory control elements for the CLA-1 gene was fused to a firefly luciferase reporter gene, were grown in above-men- 
tioned growth medium with $600 \mu \mathrm{g} / \mathrm{ml} \mathrm{G} 418$.

Transcriptional Activity Assay of CLA-1 Using CLApLUC HepG2 Cells The CLAp-LUC HepG2 cells were seeded in 96-well plates at 50000 cells/well in $100 \mu \mathrm{l}$ media. After incubation, the cells at $80 \%$ confluence were washed once with PBS $(137 \mathrm{~mm} \mathrm{NaCl}, 2.7 \mathrm{~mm} \mathrm{KCl}, 4.3 \mathrm{~mm}$ $\mathrm{Na}_{2} \mathrm{HPO}_{4}, 1.4 \mathrm{~mm} \mathrm{KH} \mathrm{PO}_{4}[\mathrm{pH} 7.3]$ ), followed by refeeding with $200 \mu \mathrm{l}$ MEM-EBSS media containing the indicated concentrations of test samples or vehicle $(0.1 \%$ dimethyl sulfoxide (DMSO)). After the indicated time of incubation at $37^{\circ} \mathrm{C}$, cells were washed with phosphate buffered solution (PBS), and then the luciferase activity was detected as relative luminescence units (RLUs) using the Luciferase Assay System (Promega, Madison, WI, U.S.A.).

RNA Isolation and Real Time Reverse Transcriptase (RT)-Polymerase Chain Reaction (PCR) Evaluation of CLA-1 transcriptional level by RNA isolation and real time reverse transcriptase (RT)-PCR was conducted as described previously. ${ }^{11)}$ Briefly, HepG2 cells were plated in a 6-well plate in MEM-EBSS media with $10 \%$ FBS at 500000 cells/well and incubated for $24 \mathrm{~h}$. After another $24 \mathrm{~h}$ treatment with the indicated concentration of pratensein or vehicle $\left(0.1 \%\right.$ DMSO) at $37^{\circ} \mathrm{C}$, total cellular RNA was isolated using QuickPrep Total RNA Extraction Kit (GE Healthcare, Piscataway, NJ, U.S.A.), and analyzed by agarose gel electrophoresis to confirm that ribosomal RNAs were intact. RNA samples $(4 \mu \mathrm{g})$ were reverse transcribed using SuperScript III First-Strand Synthesis System (Invitrogen, Carlsbad, CA, U.S.A.) and random hexamers according to the manufacturer's instructions. For quantitative real time PCR, SYBR Green PCR Core Reagent (Applied Biosystems, Foster City, CA, U.S.A.) was used on an iQ5 Multicolor RealTime PCR Detection System (Bio-Rad, California, U.S.A.). The sequences of the forward and reverse primers used for amplification were 5'-CTG GCA GAA GCG GTG ACT-3' and 5'-CAG AGC AGT TCA TGG GGA TT-3' for CLA-1 (the size of the amplicon is $97 \mathrm{bp}$ ), 5'-TCC ACT GGC GTC TTC ACC-3' and 5'-GGC AGA GAT GAT GAC CCT TTT$3^{\prime}$ for human GAPDH (the size of the amplicon is $78 \mathrm{bp}$ ). The PCR reaction conditions were $95^{\circ} \mathrm{C}$ for $5 \mathrm{~min}$ followed by 40 cycles of $95^{\circ} \mathrm{C}$ for $15 \mathrm{~s}$ and $60^{\circ} \mathrm{C}$ for $30 \mathrm{~s}$. A step of $78^{\circ} \mathrm{C}$ for $10 \mathrm{~s}$ during which fluorescence was measured was included at the end of each cycle. The reactions were subjected to a heat dissociation protocol after the final cycle of PCR to indicate the proper temperature for fluorescence detection. After PCR amplifications, data were analyzed with iQ5 Optical System version 1.1.1442.0 software (Bio-Rad). The threshold cycle $(\mathrm{Ct})$ was calculated from the programme. Serial dilution of the cDNA was subjected to real time PCR. For each transcript, plots of $\log _{10}$ (dilution factor) against the $\mathrm{Ct}$ values provided an estimate of the efficiency of the amplification. The relative amounts of CLA-1 transcript in different samples were calculated according to the Pfaffl's method. ${ }^{12)}$

Analysis of Cell Surface CLA-1 Expression by Flow Cytometry or Western Blot For the flow cytometry assays, the pretreatment of cells was the same as that for RNA isolation. Then HepG 2 cells were trypsinized from the plate, washed and resuspended in $4 \%$ paraform fixing solution, and then incubated overnight at $4{ }^{\circ} \mathrm{C}$. After the fixation, cells were blocked for $15 \mathrm{~min}$ at $4{ }^{\circ} \mathrm{C}$ in PBS containing 5\% FBS.
Cells were then incubated with monoclonal antibody to CLA-1 (BD Biosciences, San Jose, CA, U.S.A.) at a final dilution of $1: 50\left(4{ }^{\circ} \mathrm{C}, 1 \mathrm{~h}\right)$, followed by washing and staining cells with fluorescein isothiocyanate (FITC)-conjugated goat antibody to mouse immunoglobulin (Ig)G (Santa Cruz Biotechnology, Santa Cruz, CA, U.S.A.) (1:100 dilution, $\left.4^{\circ} \mathrm{C}, 1 \mathrm{~h}\right)$. The cell suspension was centrifuged $(800 \times \boldsymbol{g}$, $\left.3 \mathrm{~min}, \quad 4^{\circ} \mathrm{C}\right)$, the obtained pellet was resuspended in PBS, and fluorescence intensity was analyzed using an Epics XL flow cytometer (Coulter Corporation, Miami, FL, U.S.A.). Western blot assay of CLA-1 expression in HepG2 cells was performed as described previously. ${ }^{8}$

Analysis of Cellular Uptake of DiI-Labeled HDL by Flow Cytometry Cellular uptake of DiI-HDL by HepG2 was analyzed by flow cytometry as described previously. ${ }^{11)}$ Briefly, HepG2 cells were plated in 24-well plates at 50000 cells/well, followed by being treated for about $18 \mathrm{~h}$ with $2.08 \mu \mathrm{M}$ pratensein, and then incubated with DiI-HDL $(2 \mu \mathrm{g} / \mathrm{ml})$ for $4 \mathrm{~h}$ at $37^{\circ} \mathrm{C}$. Competition control cells were simultaneously incubated with DiI-HDL and 40-fold excess of unlabeled HDL. Cells were then washed with PBS and incubated with PBS containing $0.5 \%$ bovine serum albumin (BSA) and $2 \mathrm{~mm}$ ethylenediamine tetraacetic acid (EDTA) for $1 \mathrm{~h}$ at $4{ }^{\circ} \mathrm{C}$, and detached from the plate by gentle pipetting. The cell suspension was centrifuged $(800 \times \boldsymbol{g}, 3 \mathrm{~min}$, $4{ }^{\circ} \mathrm{C}$ ), the obtained pellet was resuspended in PBS, and DiI fluorescence was analyzed using a BD FACSCalibur flow cytometer (BD Biosciences, San Jose, CA, U.S.A.).

Luciferase Reporter Assay The reporter constructs contained the CLA-1 upstream regulatory sequence ${ }^{3)}$ spanning the region from -1195 to $-62 \mathrm{bp}$ (nucleotide +1 corresponds to the A of the ATG start codon, the same as below). pGL3-CLAp1 ( -1195 to $-62 \mathrm{bp})$, pGL3-CLAp $(-1055$ to $-62 \mathrm{bp})$, pGL3-CLAp3 (-419 to $-62 \mathrm{bp})$, pGL3-CLAp4 (-232 to $-62 \mathrm{bp})$ and pGL3-CLAp5 (-182 to $-62 \mathrm{bp})^{11)}$ were purified using PureYield ${ }^{\mathrm{TM}}$ Plasmid Midiprep System (Promega). The reporter plasmid $(0.2 \mu \mathrm{g})$ was transfected into $50000 \mathrm{HepG} 2$ cells respectively using cationic liposome transfection method (Lipofectamine 2000, Invitrogen). Cells were cotransfected with $0.02 \mu \mathrm{g}$ of the pRL-TK vector (Promega) to normalize differences in transfection efficiency. To detect the pratensein induction of different constructs, HepG2 cells were incubated with $2.08 \mu \mathrm{M}$ pratensein or vehicle $(0.1 \%$ DMSO) in MEM medium without FBS $6 \mathrm{~h}$ after transfection. Cells were harvested $18 \mathrm{~h}$ later, and the luciferase activity was detected as relative luminescence units (RLUs) using the Luciferase Assay System (Promega).

Data Analysis and Statistical Analysis Apparent $\mathrm{EC}_{50}$ values were calculated using SigmaPlot 9.0 (San Jose, CA, U.S.A.). All experiments were performed in triplicate, and representative results are presented. Quantitative data are expressed as mean \pm S.D. Statistical significance of the data was evaluated by Student's $t$-test. Differences were considered significant at a $p<0.05$.

\section{RESULTS}

Effect of Isoflavone Compounds on CLA-1 Promoter Activity In the previous study, we identified that pratensein increased the luciferase activity of CLAp-LUC HepG2 cells 
(A)

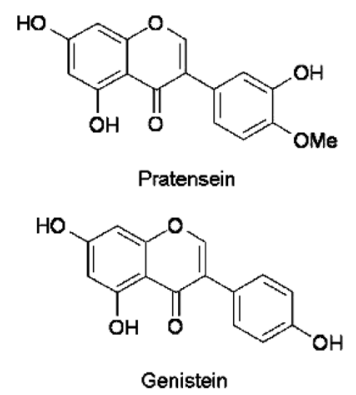

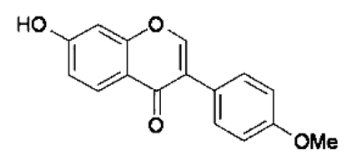

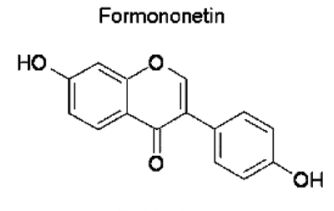

Daidzein

(B)

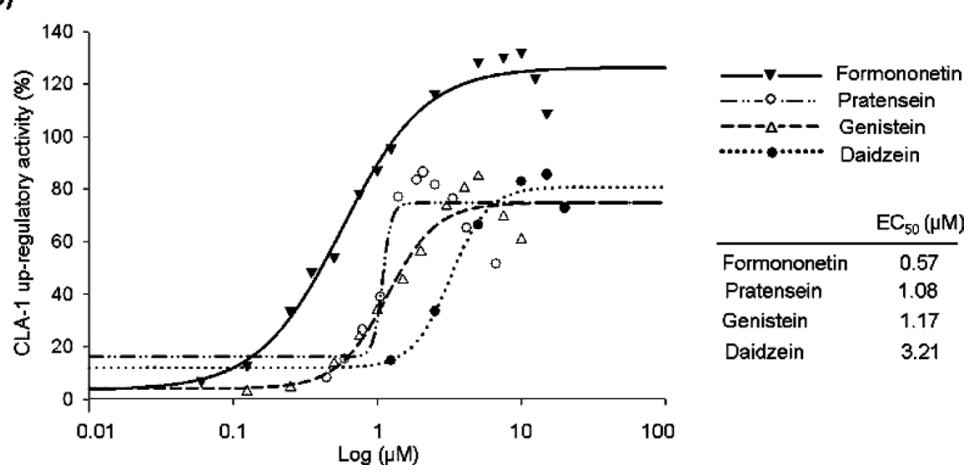

Fig. 1. Structures of Four Isoflavones and Their Activity in Cell-Based Model for CLA-1 Up-Regulators

(A) The structures of four isoflavones. (B) The effects of four isoflavones on CLA-1 transcriptional activity in cell-based model for CLA-1 up-regulators. The CLAp-LUC HepG2 cells were seeded in 96 -well plates at 50000 cells/well for about $6 \mathrm{~h}$, followed by substitution with $200 \mu 1$ medium containing serial dilution of pratensein, formononetin, genistein and daidzein. Eighteen hours later, the cells were harvested for measurements of luciferase activity. Each sample was at least triplicated and quantitative data are expressed as mean.

with a measured $\mathrm{EC}_{50}$ of $1.08 \mu \mathrm{M} .^{8)}$ To investigate if the upregulation of CLA-1 promoter activity by pratensein is due to its isoflavone structure, other three isoflavone compounds, formononetin, genistein and daidzein which are main components of $T$. pratense L., were also tested using CLAp-LUC HepG2 cells. The results showed that these isoflavone compounds enhanced the transcriptional activity of CLA-1 in a similar does-dependent manner, and their measured $\mathrm{EC}_{50}$ value were $0.57 \mu \mathrm{M}, 1.17 \mu \mathrm{M}$ and $3.21 \mu \mathrm{M}$, respectively. The maximal CLA-1 up-regulatory activity of formononetin reached $132 \%$ at about $10 \mu \mathrm{M}$, genistein reached $85 \%$ at about $5 \mu \mathrm{M}$, and daidzein reached $80 \%$ at about $15 \mu \mathrm{M}$ (Fig. 1).

Influence of Pratensein on CLA-1 mRNA Level To confirm that the CLA-1 expression level was increased by pratensein due to the up-regulation of transcriptional activity, quantitative real time RT-PCR was performed to investigate the abundance of CLA-1 mRNA in HepG2 cells with and without pratensein treatment. As illustrated in Fig. 2A, CLA1 mRNA level was significantly up-regulated by $80.5 \%$ at $2.08 \mu \mathrm{M}$ pratensein.

Influence of Pratensein on CLA-1 Protein Level To determine if the increase in CLA-1 mRNA level resulted in the induction of CLA-1 protein level, flow cytometry analysis of cell surface expressed CLA-1 was conducted using HepG2 cells with and without pratensein treatment. An induction of CLA-1 was observed when HepG2 cells were incubated with $2.08 \mu \mathrm{M}$ pratensein. The cell surface protein level of CLA-1 was increased by $41.6 \%$ (Fig. 2B). Furthermore, the CLA-1 protein level was measured in HepG2 cells with and without different concentration of pratensein, genistein and formononetin treatment by Western blotting (Fig.
2C). The result showed that these isoflavones stimulated the protein level of CLA-1 dose-dependently. After treatment of HepG2 cells with $5 \mu \mathrm{M}$ pratensein, genistein and formononetin, the protein level of CLA-1 was significantly increased by about $47.5 \%, 33.2 \%$ and $84.8 \%$, respectively.

Influence of Pratensein on the Uptake of DiI-HDL in HepG2 Cells To test whether pratensein enhanced the selective uptake of lipids from HDL by increasing the expression of CLA-1, fluorescence-labeled DiI-HDL uptake by HepG2 cells was measured in the presence or absence of $2.08 \mu \mathrm{M}$ pratensein. The result showed that $2.08 \mu \mathrm{M}$ pratensein promoted the uptake of DiI-HDL into the HepG 2 cells by $82.1 \%$, and the increase was neutralized by 40 -fold excess of unlabeled HDL (Fig. 3).

Influence of Pratensein on Different cis-Elements of CLA-1 According to the previous reports, several functional cis-regulatory elements in the promoter region of CLA-1 play important roles in its transcriptional regulation, including LXRE ( -1124 to $-1139 \mathrm{bp}),{ }^{13)}$ PPRE $(-459$ to $-472 \mathrm{bp}){ }^{14)} \mathrm{SRE}(-288$ to $-297 \mathrm{bp}$ ) and adjacent Sp1-binding sites, ${ }^{15)}$ LRH-1 or SF1 responsive element $(-204$ to $-224 \mathrm{bp}$ ), ${ }^{3,16)}$ etc. (Fig. 4A). In order to determine putative pratensein responsive region in the CLA-1 promoter, five different promoter-fragment/luciferase reporter constructs with progressive $5^{\prime}$ end deletion were transiently expressed in HepG2 cells. As previously reported, ${ }^{11)}$ the basal promoter activity decreased markedly upon removal of the bases between -1055 and $-419 \mathrm{bp}$. Removal of the bases from -419 to $-232 \mathrm{bp}$ reduced the promoter activity further (Fig. 4B). The induction of the promoter activity of these constructs by pratensein was detected. The decrease of up-regulatory luciferase activity in pGL3-CLAp3, pGL3-CLAp4 and 
(A)

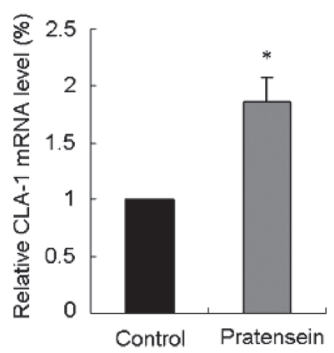

(B)

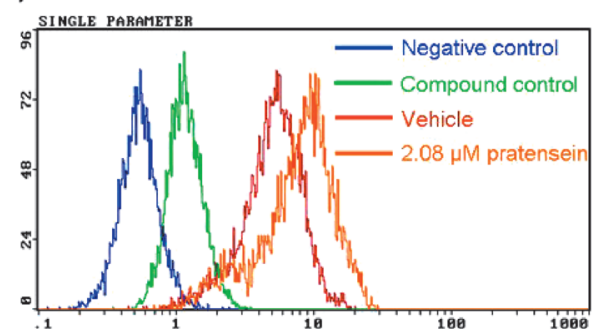

(C)
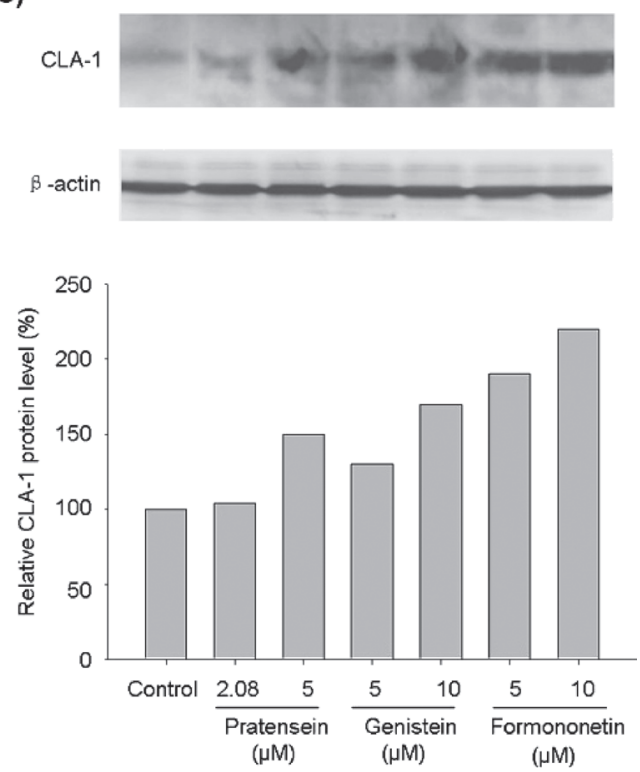

Fig. 2. Effects of Pratensein on Expression of CLA-1 in HepG2 Cells

(A) Pratensein stimulated CLA-1 transcription. The CLA-1 mRNA level was detected using real time RT-PCR analysis. Values represent the mean \pm S.D. of three independent experiments. The asterisk denotes a significant difference $(* p<0.05)$. (B) Pratensein increased CLA-1 protein level in HepG2 cells. The cell surface CLA-1 protein level was detected using flow cytometry analysis. Negative control cells were neither treated by pratensein nor incubated with primary antibody. Compound control cells were treated by $2.08 \mu \mathrm{M}$ pratensein but not incubated with primary antibody. Vehicle cells were not treated by pratensein before being incubated with primary antibody. (C) CLA-1 protein level was detected using Western blot analysis. A representative immunoblot is shown upside of a graph, showing the abundance of the immunoreactive CLA-1 normalized to $\beta$-actin.

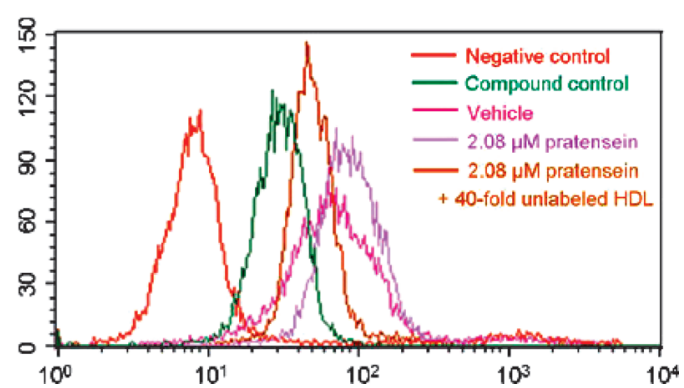

Fig. 3. Flow Cytometry Analysis for Uptake of DiI-HDL by HepG2 Cells

HepG2 cells were treated by indicated concentration of pratensein, and the uptake of DiI-HDL was measured by flow cytometry as described in Materials and Methods. Negative control cells were neither treated by pratensein nor incubated with DiI-HDL. Compound control cells were treated by $2.08 \mu \mathrm{M}$ pratensein but not incubated with DiIHDL. Vehicle cells were not treated by pratensein before being incubated with $2 \mu \mathrm{g} / \mathrm{ml}$ DiI-HDL. Competition control cells were simultaneously incubated with DiI-HDL and 40 -fold excess of unlabeled HDL.

pGL3-CLAp5 transfected HepG2 cells were significant $(p<0.01)$, indicating the existence of important regulatory elements within -1055 to $-182 \mathrm{bp}$. These regulatory elements may be PPRE and/or SRE and/or LRH-1 RE, or other elements which has not been found/reported (Fig. 4C).

\section{DISCUSSION}

In the previous study, pratensein was shown to up-regulate CLA-1 transcription in cell-based high-throughput screening model for CLA-1 up-regulators. ${ }^{8)}$ Here, we found that other isoflavones such as formononetin, genistein and daidzein also significantly up-regulated CLA-1 transcriptional activity in this cell-based screening model. The rank order of up-regulatory activity was formononetin $>$ pratensein $>$ genistein $>$ daidzein. In addition, the dose-effect curve of pratensein is the steepest in all of curves. The reason for differences in
(A)

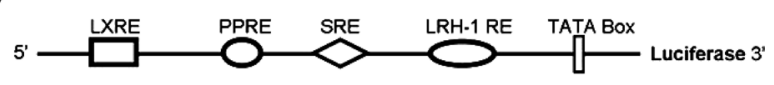

(B)

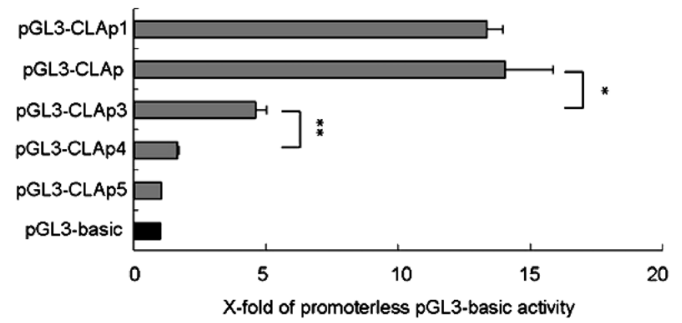

(C)

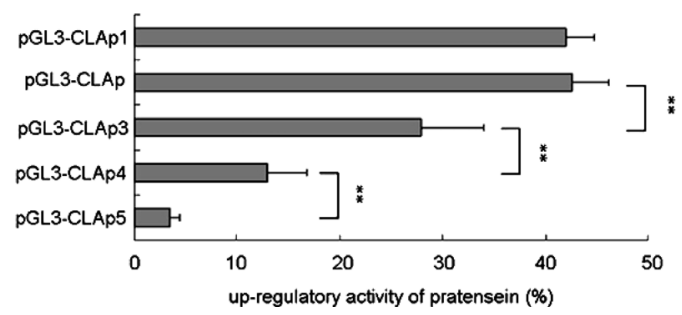

Fig. 4. Characterization of DNA Motifs Mediating Pratensein Up-Regulation of CLA-1 Promoter Activity

(A) Schematic of CLA-1 promoter region containing functional cis-regulatory elements. (B) The relative luciferase activity of different constructs in transiently transfected HepG2 cells. (C) Deletion analysis of CLA-1 promoter mapped the pratenseinresponsive region. HepG2 cells were transiently transfected with different constructs and treated with $2.08 \mu \mathrm{M}$ pratensein. The average of five separate experiments performed in duplicate is shown as mean \pm S.D. The asterisks denote a significant difference $(* p<0.05, * * p<0.01)$.

$\mathrm{EC}_{50}$ and slope of the curves of four isoflavones may be related to their minor differences in their chemical structures such as the number and the position of hydroxyl groups. For example, the chemical structure differences may influence the affinity of different compounds binding to potential transcriptional factors which regulate the CLA-1 transcription. 
However, genistin, a glycoside derived from genistein, did not affect CLA-1 transcriptional activity in a concentration range of $1-40 \mu \mathrm{M}$ (data not shown). This result suggests that forms of isoflavones (aglycones vs. glycosides) are more important because they directly affect the bioavailability and thus the bioactivity of isoflavones. Consistent with this, genistein and daidzein, but not their glycosides, are readily transported across human intestinal epithelial cell monolayers. ${ }^{17)}$ The aglycones, for these reasons, are often considered the active form of the isoflavones.

CLA-1/SR-BI has been identified to be responsible for hepatic HDL-associated cholesterol uptake independent of the classic LDL pathway. The enhancement of CLA-1/SR-BI expression and/or its activity represents a significantly potential strategy to promote reverse cholesterol transport (RCT) and to inhibit atherosclerosis. ${ }^{18,19)}$ Studies have suggested that CLA-1/SR-BI expression in the liver is especially important for HDL metabolism and overall cholesterol homeostasis in vivo. ${ }^{20)}$ Furthermore, the hepatic expression of SR-BI was demonstrated as a positive regulator of macrophage RCT in vivo. ${ }^{21)}$ A human hepatoma cell line (HepG2) was used to evaluate the effect of pratensein on CLA-1 expression in the present work. It was confirmed that both mRNA and protein level of CLA-1 were increased in pratensein treated HepG2 cells (Figs. 2A, B). The up-regulated CLA-1 was functional in HepG2 cells by increasing the uptake of DiI-HDL (Fig. 3), which is considered as a reliable surrogate for SR-BI-dependent selective uptake of lipids from HDL. ${ }^{1,22}$ Furthermore, the increasing effect can be neutralized by 40 -fold excess of unlabeled HDL. These results suggest that pratensein may stimulate the CLA-1 expression in hepatocytes, which plays a pivotal role in the final step of RCT.

The weak estrogen-like properties of isoflavonoids were discovered over 50 years. In recent years, the overall effects of the isoflavones on human health have been the subject of lively debate largely based on their presumed estrogenic properties. However, missing from our knowledge base is the systematic identification of the cellular and biochemical targets of isoflavones and the mechanisms that they influence atherosclerosis. T. pratense L. is an important kind of legume plant which is widely grown in China and other countries in the world. The content of isoflavone in T. pratense is as high as $1-2 \%$ on the basis of dry weight. Red clover botanical dietary supplements have received much attention recently for their potential use in the treatment of menopause symptoms, maintenance/improvement of bone and cardiovascular health, and reported benign effects on the breast and endometrium (see Booth et $a l^{23}$ ) and references therein). Formononetin was reported to be one of the predominant isoflavones in all parts of red clover ( $T$. pratense L.), along with some other minor aglycones, including daidzein, genistein, pratensein, etc. ${ }^{24)}$ The previous studies have not illuminated the exact mechanism(s) of isoflavones including pratensein. To the best of our knowledge, this is the first report that pratensein may act as an effective up-regulator of CLA-1 expression in hepatocytes.

In this study, the cis-elements responsible for the pratensein up-regulatory effects were mapped to the -1055 / -182 bp fragment of CLA-1 promoter in HepG2 cells (Fig. 4), containing known functional PPRE, SRE, LRH-1 RE and other known or unknown cis-element(s). The promoter se- quences of both human and murine SR-BI genes are very $G C$ rich. $^{3,25)}$ Tréguier et al. ${ }^{15)}$ reported that the promoter region of CLA-1/SR-BI is transactivated by transcription factor sterol regulatory element binding protein 2 (SREBP-2), a key regulator of cellular cholesterol homeostasis. A functional SRE was identified within $-288 \mathrm{bp}$ and $-297 \mathrm{bp}$, adjacent to an Sp1-binding site. ${ }^{14)}$ Mullen et al. ${ }^{26)}$ reported that in HepG2 cells, isoflavone-containing soy extract and individual isoflavone increased the mature form of SREBP-2. It is also known that isoflavones may affect other pathway involved in lipid and sterol metabolism. The study of Dang et $a l .{ }^{27)}$ show peroxisome proliferator-activated recetor (PPAR) $\gamma$ is a molecular target for genistein, and at the micromolar range, genistein binds to and transactivates PPAR $\gamma$, leading to a decrease of osteogenesis and an increase in adipogenesis. Likewise, this is consistent with a study reporting genistein and daidzein activated PPAR $\alpha$ and PPAR $\gamma$ in transfected RAW 264.7 cells. $^{28)}$ Unlike the highly specific estrogen receptors, PPARs bind a wide number of ligands and directly affect lipid metabolism by enhancing transcription of PPAR-regulated genes. Consistent with these previous reports that isoflavones may act on multiple pathways, our results suggest that pratensein up-regulate CLA-1 promoter through more than one cis-element.

In summary, our results show for the first time that pratensein is a novel up-regulator of CLA-1 expression in hepatocytes. Moreover, we also find that pratensein may up-regulate CLA-1 promoter through more than one cis-element such as PPRE, SRE and others. Further investigation is required to delineate whether pratensein may facilitate reverse cholesterol transport (RCT) and exert favorable effects in the peripheral cells and in vivo. Nevertheless, our study may provide new insight into the therapeutic value of pratensein in the treatment of atherosclerosis.

Acknowledgements This work was supported by $\mathrm{Na}$ tional Natural Science Foundation of China (Grant No. 90813027) and National Commonweal Research Institute Foundation (Grant No. IMBF 20060201).

\section{REFERENCES}

1) Acton S., Rigotti A., Landschulz K. T., Xu S., Hobbs H. H., Krieger M., Science, 271, 518-520 (1996).

2) Calvo D., Dopazo J., Vega M. A., Genomics, 25, 100-106 (1995).

3) Cao G. Q., Garcia C. K., Wyne K. L., Schultz R. A., Parker K. L., Hobbs H. H., J. Biol. Chem., 272, 33068-33076 (1997).

4) Ji Y., Jian B., Wang N., Sun Y., Moya M. L., Phillips M. C., Rothblat G. H., Swaney J. B., Tall A. R., J. Biol. Chem., 272, 20982-20985 (1997).

5) Anthony M. S., Clarkson T. B., Williams J. K., Am. J. Clin. Nutr., 68, 1390S-1393S (1998).

6) Ososki A. L., Kennelly E. J., Phytother. Res., 17, 845-869 (2003)

7) Cassidy A., de Pascual Tereas S., Rimbach G., Expert Rev. Mol. Med., 5, 1-15 (2003).

8) Yuan Y., Zhang Z., Jiang W., Gao L., Zhao G., Zheng Z., Wang M., Si S., Hong B., J. Biomol. Screen., 12, $211-219$ (2007).

9) Cassidy A., Albertazzi P., Lise Nielsen L., Hall W., Williamson G., Teten L., Atkin S., Cross H., Manios Y., Wolk A., Steiner C., Branca F., Proc. Nutr. Soc., 65, 76-92 (2006).

10) Sharma R. D., Atherosclerosis, 33, 371-375 (1979).

11) Bao Y., Yang Y., Wang L., Gao L., Jiang W., Wang L., Si S., Hong B., Atherosclerosis, 204, 127-135 (2009).

12) Pfaffl M. W., Nucleic Acids Res., 29, e45 (2001).

13) Malerød L., Juvet L. K., Hanssen-Bauer A., Eskild W., Berg T., 
Biochem. Biophys. Res. Commun., 299, 916-923 (2002).

14) Malerød L., Sporstøl M., Juvet L. K., Mousavi A., Gjøen T., Berg T., Biochem. Biophys. Res. Commun., 305, 557-565 (2003).

15) Tréguier M., Doucet C., Moreau M., Dachet C., Thillet J., Chapman M. J., Huby T., Arterioscler. Thromb. Vasc. Biol., 24, 2358-2364 (2004).

16) Schoonjans K., Annicotte J. S., Huby T., Botrugno O. A., Fayard E., Ueda Y., Chapman J., Auwerx J., EMBO Rep., 3, 1181-1187 (2002).

17) Steensma A., Noteborn H. P. J. M., Jagt R. C. M. van der, Polman Th. H. G., Mengelers M. J. B., Kuiper H. A., Environ. Toxicol. Pharmacol., 7, 209-212 (1999).

18) Acton S. L., Kozarsky K. F., Rigotti A., Mol. Med. Today, 5, 518-524 (1999).

19) Price M. J., Shah P. K., Rev. Cardiovasc. Med., 3, 129-137 (2002).

20) Trigatti B., Covey S., Rizvi A., Biochem. Soc. Trans., 32, 116-120 (2004).
21) Zhang Y., Da Silva J. R., Reilly M., Billheimer J. T., Rothblat G. H., Rader D. J., J. Clin. Inves., 115, 2870-2874 (2005).

22) Nieland T. J., Penman M., Dori L., Krieger M., Kirchhausen T., Proc. Natl. Acad. Sci. U.S.A., 99, 15422-15427 (2002).

23) Booth N. L., Piersen C. E., Banuvar S., Geller S. E., Shulman L. P., Farnsworth N. R., Menopause, 13, 251—264 (2006).

24) Tsao R., Papadopoulos Y., Yang R., Young J. C., McRae K., J. Agric. Chem., 54, 5797-5805 (2006).

25) Mizutani T., Yamada K., Minegishi T., Miyamoto K., J. Biol. Chem., 275, 22512-22519 (2000).

26) Mullen E., Brown R. M., Osborne T. F., Shay N. F., J. Nutr., 134, 2942-2947 (2004).

27) Dang Z. C., Audinot V., Papapoulos S. E., Boutin J. A., Löwik C. W., J. Biol. Chem., 278, 962-967 (2003).

28) Mezei O., Banz W. J., Steger R. W., Peluso M. R., Winters T. A., Shay N., J. Nutr., 133, 1238-1243 (2003). 